\title{
PROCESOS COGNITIVOS IMPLICADOS EN LA LECTURA Y ESCRITURA DE NIÑOS Y NIÑAS DEL TERCER GRADO DE EDUCACIÓN PRIMARIA RESIDENTES EN LIMA Y PIURA
}

\author{
COGNITIVE PROCESSES INVOLVED IN READING AND WRITING FOR CHILDREN OF \\ THIRD GRADE OF ELEMENTARY SCHOOL IN LIMA AND PIURA \\ Alejandro Dioses Ch. '; Cecilia Evangelista Z.; Adriana Basurto T.; Miluska Morales C.; Mónica Alcántara P. \\ Universidad Nacional Mayor de San Marcos, Perú \\ (RECibido El 11/01/2010, ACEPTADo El 08/06/2010.)
}

\begin{abstract}
RESUMEN
Se investigó, en escolares de tercer grado de primaria de Lima Metropolitana y Piura, si existían diferencias significativas en el desarrollo de los procesos cognitivos implicados en la lectura y escritura, considerando las variables género, nivel socioeconómico, gestión de la institución educativa y localidad de residencia. Para la recolección de los datos se adaptó previamente la Batería de Evaluación Cognitiva de Lectura y Escritura, estableciéndose su confiabilidad y validez. Los resultados indicaron diferencias en los procesos cognitivos examinados en razón de las variable, nivel socioeconómico, gestión de la institución educativa y localidad de residencia.
\end{abstract}

Palabras clave: Cognitivo; nivel léxico; procesamiento sintáctico-semántico de lectura; procesamiento sintáctico-semántico de escritura.

\begin{abstract}
It was investigated, in students of the third grade of elementary school of Metropolitan Lima and Piura, if there were significant differences in the development of cognitive processes involved in reading and writing, considering the variables of gender, socioeconomic status, school management and town of residence. To gather data, it was adapted previously a Battery of Cognitive Assessment for Reading and Writing, establishing its reliability and validity. The results indicated differences in the cognitive processes examined in view of the variables, socioeconomic status, management of the school and town of residence.
\end{abstract}

Keywords: Cognitive; lexical level; syntactic-semantic processing of reading; syntacticsemantic processing of writing.

1 Docente principal de la Facultad de Psicología de la UNMSM.

E-mail: alediosescho@hotmail.com 


\section{INTRODUCCIÓN}

Lectura y escritura son dos actividades complejas que implican un conjunto de acciones al interior de una enmarañada estructura de conocimientos, de tal manera que para alcanzar su plena dirección es indispensable que se desarrollen en forma paralela, de una parte, el reconocimiento y la producción de las palabras, lo que involucra la decodificación lectora, la codificación y el deletreo escrito; de otra, la comprensión y la producción de textos, es decir, comprensión lectora y composición escrita.

La premisa que guió el presente estudio plantea que la adquisición de la lectura y escritura forman parte del desarrollo del lenguaje, tanto en el reconocimiento de la palabra, como en la comprensión de la información escrita, de tal forma que el aprendizaje de la lectura y escritura no constituyen un fin en sí mismo, sino que son las herramientas que llevarán a la optimización del sistema lingüístico y comunicativo del individuo, con lo cual quedará expedito para acceder a otros aprendizajes.

A partir de lo anteriormente señalado, tanto la lectura, como la escritura han de agruparse en grandes categorías para su análisis, estas son: el componente léxico, el sintáctico y el semántico (Cuetos,1990). Por ello, al leer, la primera tarea del individuo es el reconocimiento visual de los símbolos como palabras; luego, la comprensión de las relaciones entre las palabras, su orden y estructura subyacente; para posteriormente, integrar el significado de las frases en un todo, respondiendo a las exigencias que le plantea el sistema semántico. Sobre el particular, Jusi y Carpenter, en el año 1987 (Defior, 2000), comprobaron que esta serie de procesos psicolingüísticos colaboran tanto en la comprensión, como en la producción del lenguaje escrito, aunque cada una de estas facetas de la actividad lingüística tiene sus peculiaridades.

Esta visión psicolingüística con respecto al tema rápidamente se relacionó con los planteamientos efectuados por la psicología cognitiva, dando lugar a una psicolingüística cognitiva de la lengua escrita orientada al análisis del aprendizaje de la lectura y escritura desde la óptica del procesamiento de la información, tal como lo señala Valle (Defior, 2000) y que se ha convertido en la principal perspectiva de análisis hasta el momento.

Así, desde el punto de vista de la psicolingüística cognitiva, la lectura configura un proceso complejo que conlleva la construcción de una representación mental del significado del texto, la misma que no puede limitarse a la simple percepción de grafías, dado que su esencia está referida a la transformación de determinados símbolos lingüísticos en significados, a través de un proceso que transita del lenguaje al pensamiento (Dioses, 2009); sin embargo, tal como lo precisaban García Madruga y Luque hace más de una década (García; Gonzáles, 2000), lo único establecido en este proceso es que da comienzo en la percepción de un conjunto de patrones gráficos, siendo la tarea de los investigadores determinar de la manera más exacta los tipos de representaciones mentales que se suscitan, las transformaciones intermedias y los procesos que confluyen para alcanzar esa representación mental.

De otra parte, la investigación acerca de escritura, desde la visión de la psicología cognitiva, tiene sus orígenes tanto en los trabajos de Flower y Hayes (1980), como en los de Bereiter y Scardamalia (1987), quienes analizaron la composición escrita, identificando y describiendo los procesos implicados en ella, además de establecer cómo se produce el tránsito de escritor novato a escritor experto. 
Resultados de posteriores investigaciones (Morles, 1999; Tapia, 1999) confirman que en la escritura intervienen varios procesos, entre los que destacan, principalmente, la planificación, la transcripción del texto y la revisión, procesos que no necesariamente se ejecutan en un orden particular, por lo que en la actualidad se les conoce como modelos de procesamiento global (Cuetos, 1991:20).

Por ello, así descrita la situación, la problemática se orienta a describir y entender los procesos cognitivos que realiza el individuo durante la lectura y escritura para lograr la comprensión y producción de un texto, por lo que la presente investigación, asumiendo los planteamientos de Galve (2007), apuntó a ese fin.

Lo anteriormente señalado desencadenó el planteamiento de las siguientes hipótesis:

La comparación de la puntuación promedio en lectura de estudiantes de 3.er grado de

$\mathrm{H}_{1}$ : primaria residentes en Lima Metropolitana mostrará diferencias significativas según la variable sexo.

La comparación de la puntuación promedio en lectura de estudiantes de 3.er grado de

$\mathrm{H}_{2}$ : primaria residentes en Lima Metropolitana mostrará diferencias significativas según la variable nivel socioeconómico.

La comparación de la puntuación promedio en lectura de estudiantes de 3.er grado de

$\mathrm{H}_{3}$ : primaria residentes en Lima Metropolitana mostrará diferencias significativas con respecto a la variable gestión de la institución educativa a la que asisten.

$\mathrm{H}_{4}$ : El rendimiento en lectura de niños y niñas del 3.er grado de primaria de Lima Metropolitana mostrará diferencias significativas con respecto al de sus pares de Piura.

$\mathbf{h}_{\mathbf{1}}$ : El nivel léxico de niños y niñas del 3.er grado de primaria de Lima Metropolitana mostrarán diferencias significativas con respecto al nivel presentado por sus pares de Piura.

$\mathbf{h}_{2}$ : Los niveles sintáctico-semántico en lectura de oraciones de niños y niñas del 3.er grado pertenecientes a instituciones educativas de Lima Metropolitana mostrarán diferencias significativas con respecto al nivel alcanzado por el de sus pares de Piura.

$\mathbf{h}_{3}$ : Los niveles sintáctico-semántico en lectura de textos de niños y niñas del 3.er grado de Lima Metropolitana mostrarán diferencias significativas con respecto al nivel de sus pares de Piura.

$\mathrm{H}_{5}$ : El rendimiento en escritura de niños y niñas del 3.er grado de primaria de Lima Metropolitana mostrará diferencias significativas con respecto al de sus pares de Piura.

$\mathbf{h}_{\mathbf{1}}$ : El nivel léxico de niños y niñas del 3.er grado de primaria de Lima Metropolitana mostrará diferencias significativas con respecto al nivel alcanzado por sus pares de Piura.

$\mathbf{h}_{2}$ : Los niveles sintáctico-semántico en escritura de oraciones de niños y niñas del 3.er grado de primaria de Lima Metropolitana mostrarán diferencias significativas con respecto al nivel logrado por sus pares de Piura.

$\mathbf{h}_{3}$ : Los niveles sintáctico-semántico en estrategias de escritura de textos de niños y niñas del 3.er grado de primaria de Lima Metropolitana mostrarán diferencias significativas con respecto al nivel logrado por sus pares de Piura. 


\section{MATERIAL Y MÉTODO}

\section{Método}

El método utilizado para el presente estudio fue el descriptivo (Ugarriza, 1998:28), en tanto el énfasis de la investigación se orientó a la recolección, descripción e interpretación sistemática de un conjunto de datos en la variable estudiada: procesos cognitivos implicados en la lectura y escritura; sin efectuar manipulación alguna de la misma. El diseño utilizado fue el transversal-comparativo (Hernández; Fernández; Baptista, 1999).

\section{Variables}

Las variables independientes estuvieron definidas por el género, nivel socioeconómico, gestión de la institución educativa y ciudad ubicación de la institución educativa: Lima o Piura; mientras que la variable dependiente la constituyeron los procesos implicados en la lectura: nivel léxico, nivel sintáctico-semántico en la lectura de oraciones y nivel sintáctico-semántico en la lectura de textos, y la escritura, con sus dimensiones: nivel léxico, nivel sintáctico-semántico en la escritura de oraciones, y nivel sintáctico-semántico de estrategias en la escritura de textos.

\section{Participantes}

Los sujetos de estudio fueron 559 escolares varones y mujeres de 8 años, 0 meses a 9 años, 11 meses, 29 días de edad (277 procedentes de instituciones educativas de la ciudad de Lima y 282 de instituciones educativas de la ciudad de Piura) que se encontraban cursando el tercer grado de educación primaria, los mismos que fueron seleccionados mediante un muestreo polietápico donde, para cada ciudad, se efectuaron los siguientes pasos: 1) se separó los colegios por estrato socioeconómico: segmento A, B, C, D, E; 2) e cada estrato socioeconómico se separaron los colegios en públicos y privados; 3) se seleccionó, por conglomerados, los colegios representativos de cada uno de los estratos; 4) en cada colegio elegido se seleccionaron al azar simple los alumnos varones y mujeres hasta completar las cuotas establecidas.

\section{Instrumento}

La recolección de la información se efectuó con la Batería de Evaluación Cognitiva de Lectura y Escritura (BECOLE), de José Luis Galve Manzano (2005), la misma que procede del Instituto de Orientación Psicológica con sede en Madrid y fue publicada en el 2005. Esta prueba se administra desde $3{ }^{\text {ro }}$ de primaria hasta $1{ }^{\text {ro }}$ de secundaria. La versión española presentó una fiabilidad de 0.95 obtenida mediante alfa de Cronbach y un análisis de dimensionalidad mediante análisis factorial confirmatorio, con resultados superiores a 0.30 .

\section{Procedimiento}

Para el análisis psicométrico del presente estudio se desarrolló un plan piloto de evaluación bajo un diseño psicométrico y comparativo, tal cual se planteó en el estudio original español. 
Así, se contó con una muestra de 559 sujetos de tercer grado de educación primaria pertenecientes a colegios públicos y privados de las ciudades de Lima y Piura, tal como se detalla en la tabla 1. La prueba fue aplicada solo en su primer nivel (elemental) debido a las características de la muestra y los demás objetivos de la investigación.

Tabla N. ${ }^{0}$ 1. Distribución de los sujetos por gestión de la institución educativa y género.

\begin{tabular}{ccccc}
\hline & \multicolumn{2}{c}{ Gestión educativa } & \multirow{2}{*}{ Total } \\
\cline { 3 - 4 } & & Nacional & Particular & \\
\hline \multirow{2}{*}{ Género } & Masculino & 98 & 179 & 277 \\
& Femenino & 100 & 182 & 282 \\
& Total & 198 & 361 & 559 \\
\hline
\end{tabular}

De acuerdo a la estructura original de la prueba, el análisis se realizó en dos partes, la primera correspondiente al grupo de escalas de la BECOLE que evalúa los procesos de comprensión en lectura y la segunda que corresponde al grupo de escalas para evaluar los procesos de producción en escritura.

\section{RESULTADOS}

\section{Resultados del estudio psicométrico de los procesos de lectura}

\section{Análisis de ítemes (índice de discriminación)}

Como primera parte del análisis psicométrico se recurrió al cálculo del índice de discriminación bajo la modalidad de grupos extremos. Así, los puntajes de las escalas del BECOLE fueron segmentados en dos grupos: uno con los puntajes más altos y otro con los puntajes más bajos; para comparar las medias de los puntajes de ambos grupos y corroborar la hipótesis de la existencia de diferencias significativas bajo el argumento de buscar ítemes que tengan poder de discriminación.

La tabla 2 muestra los ítemes correspondientes a las pruebas de lectura que evidenciaron diferencias significativas en los grupos extremos y que fueron, por lo tanto, eliminados de la escala.

Las pruebas “comprensión lectora" (CL), “comprensión de órdenes” (COR), "conocimiento ortográfico" (ORT) y "estructuras de comprensión lectora" (ECLD) conservaron la totalidad de sus ítemes. 
Tabla N. 2. Análisis de discriminación por ítemes.

\begin{tabular}{llc}
\hline & \multicolumn{1}{c}{ Comprensión (pruebas) } & Ítemes eliminados \\
\hline ADP & Asociación de una palabra con su definición & $1-3$ \\
ADH & Asociación de una palabra con su definición: homófonas & $7-9$ \\
CL & Comprensión lectora & Ninguno \\
COR & Comprensión de órdenes & Ninguno \\
CS & Comprensión de estructuras sintácticas & $4-5-6-7-9-12$ \\
DLX & Decisión léxica & $2-3-4-5-7-9-10-11-14-19-21-24$ \\
LDP & Lectura de palabras & $27-28-29-30-33-38-39-42-44-45$ \\
ORT & Conocimiento ortográfico & 29 \\
ECLD Estructuras de comprensión lectora (dibujos) & Ninguno \\
\hline
\end{tabular}

\section{Fiabilidad}

a) Análisis de fiabilidad del nivel elemental

Se utilizó el coeficiente alfa de Cronbach, el cual indicó el grado en que covarían los ítemes de cada prueba, ello supone un indicador de la consistencia interna de esta.

Se realizó el análisis de fiabilidad para la totalidad de la batería de lectura (la suma de los puntajes que corresponden a las subpruebas), así como para las subpruebas, excluyendo los totales. La tabla 3 muestra los estadísticos globales de los procesos de lectura.

Tabla N. ${ }^{\circ}$ 3. Análisis de fiabilidad de la lectura-BECOLE nivel elemental (global).

\begin{tabular}{cccc}
\hline \multicolumn{4}{c}{ BECOLE-nivel elemental (lectura) } \\
\hline Fiabilidad & Media & Desviación típica & Varianza \\
0.7441 & 135.29 & 26.571 & 706.025 \\
\hline
\end{tabular}

b) Fiabilidad por pruebas componentes de la batería

La tabla 4 muestra los coeficientes alfa de las pruebas que componen el área de comprensión, se estimó un mínimo de 0.68 y un máximo de 0.74 . 
Tabla N. ${ }^{\circ}$ 4. Análisis de fiabilidad de la lectura-BECOLE nivel elemental (pruebas).

\begin{tabular}{llc}
\hline & \multicolumn{1}{c}{ Comprensión (pruebas) } & Fiabilidad \\
\hline ADP & Asociación de una palabra con su definición & 0.7429 \\
ADH & Asociación de una palabra con su definición: homófonos & 0.6853 \\
CL & Comprensión lectora & 0.7454 \\
COR & Comprensión de órdenes & 0.7238 \\
CS & Comprensión de estructuras sintácticas & 0.7274 \\
DLX & Decisión léxica & 0.6938 \\
LDP & Lectura de palabras & 0.7254 \\
ORT & Conocimiento ortográfico & 0.7441 \\
ECLD & Estructuras de comprensión lectora (dibujos) & 0.7456 \\
\hline
\end{tabular}

\section{Dimensionalidad de las pruebas}

Para el estudio de la dimensionalidad de las pruebas se utilizó el análisis factorial exploratorio por el método de los componentes principales con rotación varimax. En la tabla 5 se evidencian las saturaciones en cada uno de los componentes superiores al 0.30.

Se aprecia también la matriz factorial rotada según el modelo de rotación varimax, en la que aparecen los componentes extraídos y la saturación de las pruebas de cada uno de ellos, seguida de una breve explicación del número de factores, su porcentaje de varianza explicada y la matriz de correlaciones de sus componentes.

Tabla N. ${ }^{\circ}$ 5. Análisis de la dimensionalidad de la lectura-BECOLE nivel elemental (totales).

\begin{tabular}{llcc}
\hline & \multicolumn{1}{c}{ Comprensión (pruebas) } & $\mathbf{1}$ & $\mathbf{2}$ \\
\hline ADP & Asociación de una palabra con su definición & 0.755 & \\
ADH & Asociación de una palabra con su definición: homófonos & 0.624 & \\
CL & Comprensión lectora & 0.721 & \\
COR & Comprensión de órdenes & 0.757 & 0.778 \\
CS & Comprensión de estructuras sintácticas & 0.725 & \\
ORT & Conocimiento ortográfico & 0.644 & \\
ECLD & Estructuras de comprensión lectora (dibujos) & & 0.892 \\
DLX & Decisión léxica & & 0.440 \\
LDP & Lectura de palabras & &
\end{tabular}


Se obtuvieron 2 factores que explican un $54.092 \%$ de la varianza total, siendo la correlación entre los factores de 0.303 .

El modelo factorial repite la estructura del estudio original, con modificaciones en la correspondencia de las pruebas a los factores, ello debido a las diferencias en el análisis del índice de discriminación.

La tabla 6 muestra el modelo factorial de las subpruebas de comprensión, sin incluir los totales respectivos.

Tabla N. ${ }^{0}$ 6. Análisis de dimensionalidad de la lectura-BECOLE nivel elemental (subpruebas).

\begin{tabular}{|c|c|c|c|c|c|c|c|}
\hline & Comprensión (subpruebas) & 1 & 2 & 3 & 4 & 5 & 6 \\
\hline CS COM & Comprensión de estructuras sintácticas: comparativas-coordinadas & 0.756 & & & & & \\
\hline $\mathrm{CL}$ & Comprensión lectora & 0.718 & & & & & \\
\hline COR & Comprensión de órdenes & 0.687 & & & & & \\
\hline $\mathrm{ADH}$ & Asociación de una palabra con su definición: homófonos & 0.558 & & & & & \\
\hline ORT & Conocimiento ortográfico & 0.724 & & & & & \\
\hline $\mathrm{ADP}$ & Asociación de una palabra con su definición & 0.695 & & & & & \\
\hline CS PAS & Comprensión de estructuras sintácticas: pasivas & 0.746 & & & & & \\
\hline LDP PSP & Lectura de pseudopalabras & & 0.697 & & & & \\
\hline LDP PSH & Lectura de pseudohomófonas & & 0.831 & & & & \\
\hline LDP AFAE & Lectura de palabras: alta frecuencia-alta extensión & & 0.758 & & & & \\
\hline LDP AFBE & Lectura de palabras: alta frecuencia-baja extensión & & 0.818 & & & & \\
\hline LDP BFAE & Lectura de palabras: baja frecuencia-alta extensión & & 0.711 & & & & \\
\hline LDP BFBE & Lectura de palabras: baja frecuencia-baja extensión & & 0.785 & & & & \\
\hline DLX AFAE & Decisión léxica: alta frecuencia-alta extensión & & & 0.962 & & & \\
\hline DLX AFBE & Decisión léxica: alta frecuencia-baja extensión & & & 0.961 & & & \\
\hline DLX BFAE & Decisión léxica: baja frecuencia-alta extensión & & & 0.840 & & & \\
\hline DLX BFBE & Decisión léxica: baja frecuencia-baja extensión & & & 0.954 & & & \\
\hline ECLD DE & Estructuras de comprensión lectora: descripción & & & & 0.841 & & \\
\hline ECLD PS & Estructuras de comprensión lectora: problema-solución & & & & 0.848 & & \\
\hline ECLD CA & Estructuras de comprensión lectora: causalidad & & & & 0.796 & & \\
\hline ECLD CO & Estructuras de comprensión lectora: comparación & & & & 0.681 & & \\
\hline ECLD SE & Estructuras de comprensión lectora: secuencia & & & & 0.754 & & \\
\hline DLX PSP & Decisión léxica pseudopalabras & & & & & 0.817 & \\
\hline DLX PSH & Decisión léxica pseudohomófonas & & & & & 0.734 & \\
\hline CS SUB & Comprensión de estructuras sintácticas: subordinadas & & & & & & 0.790 \\
\hline CS ACT & Comprensión de estructuras sintácticas: activas & & & & & & 0.805 \\
\hline
\end{tabular}


La tabla 7 muestra la matriz de correlaciones de los factores bajo la normalización kaiser. En este análisis se obtuvo un factor adicional a los encontrados en el estudio original.

Tabla N. ${ }^{0}$ 7. Matriz de correlaciones de componentes.

\begin{tabular}{ccccccc}
\hline Componente & $\mathbf{1}$ & $\mathbf{2}$ & $\mathbf{3}$ & $\mathbf{4}$ & $\mathbf{5}$ & $\mathbf{6}$ \\
\hline 1 & 0.634 & 0.374 & -0.284 & 0.530 & 0.265 & 0.163 \\
2 & 0.008 & 0.606 & 0.791 & 0.013 & -0.073 & 0.034 \\
3 & 0.395 & -0.698 & 0.523 & 0.275 & -0.003 & 0.086 \\
4 & 0.527 & 0.020 & -0.022 & -0.773 & 0.017 & 0.352 \\
5 & -0.243 & -0.005 & -0.073 & 0.210 & -0.428 & 0.841 \\
6 & -0.325 & -0.069 & 0.120 & -0.041 & 0.861 & 0.364 \\
\hline
\end{tabular}

Se obtuvieron 6 factores que explican un $68.875 \%$ de la varianza total.

Luego se realizaron análisis factoriales de los respectivos niveles de procesamiento para valorar el agrupamiento de las pruebas, con el objetivo de establecer si se agrupaban en función de la ruta utilizada, según el modelo teórico expuesto en el estudio original.

La tabla 8 muestra los resultados del análisis factorial de las pruebas y subpruebas que componen el nivel léxico para lectura.

Tabla N. ${ }^{\circ}$ 8. Análisis factorial de la lectura-BECOLE nivel léxico.

\begin{tabular}{lllll}
\hline & \multicolumn{1}{c}{ Comprensión (Subpruebas) } & $\mathbf{1}$ & $\mathbf{2}$ \\
\hline LDP PSP & Lectura de pseudopalabras & 0.702 & & \\
LDP PSH & Lectura de pseudohomófonas & 0.835 & & \\
LDP AFAE & Lectura de palabras: alta frecuencia-alta extensión & 0.767 & & \\
LDP AFBE & Lectura de palabras: alta frecuencia-baja extensión & 0.817 & & \\
LDP BFAE & Lectura de palabras: baja frecuencia-alta extensión & 0.718 & & \\
LDP BFBE & Lectura de palabras: baja frecuencia-baja extensión & 0.784 & & \\
DLX AFAE & Decisión léxica alta frecuencia-alta extensión & & 0.960 & \\
DLX AFBE & Decisión léxica: alta frecuencia-baja extensión & & 0.960 & \\
DLX BFAE & Decisión léxica: baja frecuencia-alta extensión & & 0.860 & \\
DLX BFBE & Decisión léxica: baja frecuencia-baja extensión & & 0.953 & 0.797 \\
DLX PSP & Decisión léxica pseudopalabras & & & 0.731 \\
DLX PSH & Decisión léxica pseudohomófonas & & & 0.678 \\
ADP & Asociación de una palabra con su definición & & & \\
ADH & Asociación de una palabra con su definición: homófonos & & & \\
ORT & Conocimiento ortográfico & & & \\
\hline
\end{tabular}


Se obtuvieron 3 factores que explican un $65.867 \%$ de la varianza total.

El modelo factorial coincide en el número de factores hallados en el estudio original y con la asignación de los elementos a cada uno de los factores, lo que se considera un indicador favorable en la estructura de este nivel.

La tabla 9 muestra las correlaciones entre los factores, bajo el método de los componentes principales y con una normalización kaiser.

Tabla N. ${ }^{0}$ 9. Matriz de correlación de componentes.

\begin{tabular}{cccc}
\hline Componente & $\mathbf{1}$ & $\mathbf{2}$ & $\mathbf{3}$ \\
\hline 1 & 0.671 & -0.490 & 0.557 \\
2 & 0.612 & 0.790 & -0.042 \\
3 & -0.419 & 0.369 & 0.829 \\
\hline
\end{tabular}

La tabla 10 muestra los resultados del análisis factorial de las pruebas y subpruebas que componen el nivel sintáctico-semántico para lectura.

Tabla N. ${ }^{0}$ 10. Análisis factorial de la lectura-BECOLE nivel sintáctico-semántico.

\begin{tabular}{llc}
\hline & \multicolumn{1}{c}{ Comprensión (subpruebas) } & $\mathbf{1}$ \\
\hline CS A & Comprensión de estructuras sintácticas: activas & 0.599 \\
CS S & Comprensión de estructuras sintácticas: subordinadas & 0.869 \\
CS C & Comprensión de estructuras sintácticas: comparativas-coordinadas & 0.845 \\
CS P & Comprensión de estructuras sintácticas: pasivas & 0.645 \\
T COR & Total comprensión de órdenes & 0.746 \\
\hline
\end{tabular}

Se obtuvo un factor que explica el $56.017 \%$ de la varianza total.

El modelo factorial difiere en el número de factores hallados con respecto al estudio original, ello debido a los resultados del análisis del índice de discriminación.

La tabla 11 muestra los resultados del análisis factorial de las pruebas y subpruebas que componen el nivel de estrategias de texto para lectura. 
Tabla N. ${ }^{\circ}$ 11. Análisis factorial de la lectura-BECOLE nivel estrategias de texto.

\begin{tabular}{llc}
\hline & \multicolumn{1}{c}{ Comprensión (subpruebas) } & $\mathbf{1}$ \\
\hline ECLD C & Estructuras de comprensión lectora: causalidad & 0.876 \\
ECLD D & Estructuras de comprensión lectora: descripción & 0.869 \\
ECLD S & Estructuras de comprensión lectora: secuencia & 0.851 \\
ECLD CO & Estructuras de comprensión lectora: comparación & 0.636 \\
ECLD P & Estructuras de comprensión lectora: problema-solución & 0.897 \\
CL & Comprensión lectora & 0.450 \\
\hline
\end{tabular}

Se obtuvo un factor que explica el $60.969 \%$ de la varianza total.

El modelo factorial coincide en el número de factores hallados en el estudio original, obteniendo coeficientes más altos, salvo en la prueba de "comprensión lectora" (CL).

\section{Resultados del estudio psicométrico de los procesos de escritura}

\section{Análisis de ítemes (índice de discriminación)}

Al igual que con los procesos de lectura, como primera parte del análisis psicométrico, se recurrió al cálculo del índice de discriminación bajo la modalidad de grupos extremos. Así, los puntajes de las escalas de la BECOLE fueron segmentados en dos grupos: uno con los puntajes más altos y otro con los puntajes más bajos; para comparar las medias de los puntajes de ambos grupos y corroborar la hipótesis de la existencia de diferencias significativas bajo el argumento de buscar ítemes que tengan poder de discriminación.

La tabla 12 muestra los ítemes correspondientes a las pruebas de escritura que evidenciaron diferencias significativas en los grupos extremos y que fueron, por lo tanto, eliminados de la escala.

Tabla N. ${ }^{0}$ 12. Análisis de discriminación por ítemes.

\begin{tabular}{llc}
\hline & Producción (Pruebas) & Ítemes eliminados \\
\hline DHP & Dictado de homófonos & $4-5-6-13$ \\
DDP & Dictado de palabras & $10-17-38$ \\
ES & Estructuras sintácticas & $13-20-21-22-23-24-25-26-27-28-29-30-31-32$ \\
ODF & Ordenación de frases & Ninguno \\
\hline
\end{tabular}




\section{Fiabilidad}

a) Análisis de fiabilidad de nivel elemental

Se utilizó el coeficiente alfa de Cronbach, el cual indicó el grado en que covarían los ítemes de cada prueba, lo que supone un indicador de la consistencia interna de esta.

Se realizó el análisis de fiabilidad para la totalidad de la batería de lectura (la suma de los puntajes que corresponden a las subpruebas), así como para las subpruebas, excluyendo los totales. La tabla 13 muestra los estadísticos globales de los procesos de producción (escritura).

Tabla N. ${ }^{\circ}$ 13. Análisis de fiabilidad de la escritura-BECOLE nivel elemental (global).

\begin{tabular}{cccc}
\hline \multicolumn{4}{c}{ Becole-nivel E (escritura) } \\
\hline Fiabilidad & Media & Desviación típica & Varianza \\
0.7725 & 43.13 & 12.346 & 152.412 \\
\hline
\end{tabular}

b) Fiabilidad por pruebas componentes de la batería

La tabla 14 muestra los coeficientes alfa de las pruebas que componen el área de producción, se estima un mínimo de 0.68 y un máximo de 0.72 (ligeramente menores a los coeficientes del estudio original).

Tabla N. ${ }^{0}$ 14. Análisis de fiabilidad de escritura-BECOLE nivel elemental (pruebas).

\begin{tabular}{llc}
\hline & Producción (pruebas) & Fiabilidad \\
\hline DHP & Dictado de homófonos & 0.6803 \\
DDP & Dictado de palabras & 0.7240 \\
ES & Estructuras sintácticas & 0.6834 \\
ODF & Ordenación de frases & 0.7254 \\
\hline
\end{tabular}

\section{Dimensionalidad de las pruebas}

Para el estudio de la dimensionalidad de las pruebas se utilizó el análisis factorial exploratorio por el método de los componentes principales con rotación varimax (variante con respecto al estudio original). En la tabla 15 se evidencian las saturaciones en cada uno de los componentes, superiores al 0.30 .

Se presenta también la matriz factorial rotada según el modelo de rotación varimax, en la que aparecen los componentes extraídos y la saturación de las pruebas de cada uno de ellos seguida de una breve explicación del número de factores, su porcentaje de varianza explicada y la matriz de correlaciones de sus componentes. 
Tabla N. ${ }^{\circ}$ 15. Análisis de dimensionalidad de la escritura-BECOLE nivel elemental (totales).

\begin{tabular}{llc}
\hline & \multicolumn{1}{c}{ Comprensión (pruebas) } & $\mathbf{1}$ \\
\hline DHP & Dictado de homófonos & 0.806 \\
DDP & Dictado de palabras & 0.848 \\
ES & Estructuras sintácticas & 0.685 \\
ODF & Ordenación de frases & 0.805 \\
ECLD & Estructuras de comprensión lectora (dibujos) & 0.7456 \\
\hline
\end{tabular}

Se obtuvo un factor que explica el $62.103 \%$ de la varianza total.

El modelo factorial repite la estructura del estudio original, con coeficientes ligeramente menores.

La tabla 16 muestra el modelo factorial de las subpruebas de producción, sin incluir los totales respectivos.

Tabla N. ${ }^{\circ}$ 16. Análisis factorial de la escritura-BECOLE nivel elemental (subpruebas).

\begin{tabular}{llll}
\hline & \multicolumn{1}{c}{ Producción (subpruebas) } & $\mathbf{1}$ & $\mathbf{2}$ \\
\hline DHP & Dictado de palabras homófonas & 0.693 & \\
DDP PSP & Dictado de palabras: pseudopalabras & 0.782 & \\
DDP PSH & Dictado de palabras: pseudohomófonas & 0.759 & \\
DDP AFAE & Dictado de palabras: alta frecuencia-alta extensión & 0.698 \\
DDP AFBE & Dictado de palabras: alta frecuencia-baja extensión & 0.804 & \\
DDP BFAE & Dictado de palabras: baja frecuencia-alta extensión & 0.688 & \\
DDP BFBE & Dictado de palabras: baja frecuencia-baja extensión & 0.77 & \\
ODF & Ordenamiento de frases & 0.644 & \\
ES ACT & Producción de estructuras sintácticas: activas & 0.430 & \\
ECLD CA & Estructuras de comprensión lectora: causalidad & & 0.840 \\
ECLD DE & Estructuras de comprensión lectora: descripción & & 0.961 \\
ECLD SE & Estructuras de comprensión lectora: secuencia & & 0.816 \\
ECLD CO & Estructuras de comprensión lectora: comparación & & 0.634 \\
ECLD PS & Estructuras de comprensión lectora: problema-solución & & 0.882 \\
ES PAS & Producción de estructuras sintácticas: pasivas & & 0.687 \\
ES SUB & Producción de estructuras sintácticas: subordinadas & & \\
ES COM & Producción de estructuras sintácticas: comparativas-coordinadas & & \\
\hline
\end{tabular}

La tabla 17 muestra la matriz de correlaciones de los factores bajo el método de los componentes principales y con normalización kaiser. En este análisis se obtuvo la misma 
cantidad de factores a los encontrados en el estudio original, con modificaciones en la correspondencia de las pruebas y subpruebas a los factores hallados.

Tabla N. ${ }^{0}$ 17. Matriz de correlación de componentes.

\begin{tabular}{cccc}
\hline Componente & $\mathbf{1}$ & $\mathbf{2}$ & $\mathbf{3}$ \\
\hline 1 & .786 & .586 & .198 \\
2 & -.596 & .803 & -.014 \\
3 & -.167 & -.107 & .980 \\
\hline
\end{tabular}

Se obtuvieron 3 factores que explican el $60.649 \%$ de la varianza total.

Luego, al igual que en la primera parte, se realizaron análisis factoriales de los respectivos niveles de procesamiento para valorar el agrupamiento de las pruebas, tratando de ver si se agrupan en función de la ruta utilizada, según el modelo teórico expuesto en el estudio original.

La tabla 18 muestra los resultados del análisis factorial para las subpruebas que componen el nivel léxico para producción.

Tabla N. ${ }^{\circ}$ 18. Análisis factorial de la escritura-BECOLE nivel léxico.

\begin{tabular}{llc}
\hline & \multicolumn{1}{c}{ Producción (subpruebas) } & $\mathbf{1}$ \\
\hline DHP & Dictado de palabras homófonas & 0.729 \\
DDP PSP & Dictado de palabras: pseudopalabras & 0.791 \\
DDP PSH & Dictado de palabras: pseudohomófonas & 0.757 \\
DDP AFAE & Dictado de palabras: alta frecuencia-alta extensión & 0.759 \\
DDP AFBE & Dictado de palabras: alta frecuencia-baja extensión & 0.810 \\
DDP BFAE & Dictado de palabras: baja frecuencia-alta extensión & 0.744 \\
DDP BFBE & Dictado de palabras: baja frecuencia-baja extensión & 0.780 \\
\hline
\end{tabular}

Se obtuvo un factor que explica el $58.919 \%$ de la varianza total.

El modelo factorial difiere del estudio original, considerando solo un factor.

La tabla 19 muestra los resultados del análisis factorial para las pruebas y subpruebas que componen el nivel sintáctico-semántico para producción. 
Tabla N. ${ }^{\circ}$ 19. Análisis factorial de la escritura-BECOLE nivel sintáctico-semántico.

\begin{tabular}{llrc}
\hline & \multicolumn{1}{c}{$\quad$ Producción (subpruebas) } & $\mathbf{1}$ & $\mathbf{2}$ \\
\cline { 3 - 4 } ES ACT & Producción de estructuras sintácticas: activas & & 0.824 \\
ES PAS & Producción de estructuras sintácticas: pasivas & \multicolumn{2}{c}{0.777} \\
ES SUB & Producción de estructuras sintácticas: subordinadas & 0.589 & \\
ES COM & Producción de estructuras sintácticas: coordinadas comparativas & 0.858 & \\
ODF & Ordenamiento de frases & 0.840 & \\
\hline
\end{tabular}

Se obtuvieron dos factores que explican el $66.071 \%$ de la varianza total.

El modelo factorial difiere del estudio original, encontrando un factor adicional. El coeficiente de correlación de ambos factores es de 0.584 .

\section{Normas}

Tras completar el análisis psicométrico del test BECOLE se ha visto conveniente diseñar una tabla de normas - baremo - general (no se hallaron diferencias significativas de acuerdo al sexo) para el nivel elemental.

Se obtuvo a partir de una muestra de 559 niños y niñas que cursaban el tercer grado de educación primaria regular en instituciones educativas públicas y privadas de las ciudades de Lima y Piura.

Tabla N. ${ }^{\circ}$ 20. Baremo BECOLE-nivel elemental.

\begin{tabular}{|c|c|c|c|c|c|c|c|c|}
\hline & \multicolumn{4}{|c|}{ Comprensión } & \multicolumn{4}{|c|}{ Producción } \\
\hline & Nivel léxico & $\begin{array}{l}\text { Nivel sintáctico } \\
\text { semántico }\end{array}$ & $\begin{array}{l}\text { Nivel estrategias } \\
\text { de texto }\end{array}$ & Total & Nivel léxico & $\begin{array}{l}\text { Nivel sintáctico } \\
\text { semántico }\end{array}$ & ECLD & Total \\
\hline Media & 93.28 & 19.93 & 17.20 & 119.37 & 33.46 & 7.72 & 559 & 41.10 \\
\hline DS & 14.57 & 7.18 & 8.99 & 23.54 & 9.34 & 3.91 & 6.53 & 12.02 \\
\hline Varianza & 212.41 & 51.68 & 80.95 & 554.35 & 87.30 & 15.35 & 5.625 & 144.61 \\
\hline \multicolumn{9}{|l|}{$\mathrm{Pc}$} \\
\hline 1 & 56 & $1-6$ & $1-2$ & 57 & $1-15$ & 1 & - & $1-17$ \\
\hline 5 & 71 & 7 & 3 & 80 & 16 & 2 & - & 18 \\
\hline 10 & 76 & 9 & 6 & 89 & 22 & 3 & - & 27 \\
\hline 15 & 79 & 11 & 8 & 95 & 25 & 4 & - & 29 \\
\hline 20 & 81 & 13 & 9 & 100 & 27 & - & 1 & 32 \\
\hline 25 & 83 & 15 & 10 & 104 & 28 & 5 & - & 35 \\
\hline 30 & 85 & 16 & 11 & 107 & 30 & - & 2 & 36 \\
\hline 35 & 88 & 18 & 13 & 110 & 31 & - & - & 37 \\
\hline 40 & 90 & 19 & 14 & 114 & 32 & 6 & 3 & 39 \\
\hline 45 & 92 & 20 & 16 & 116 & 34 & - & 4 & 41 \\
\hline 50 & 94 & 21 & 17 & 120 & 35 & 7 & 5 & 42 \\
\hline 55 & 96 & 23 & 18 & 123 & 36 & - & 6 & 44 \\
\hline 60 & 97 & 23 & 19 & 126 & 37 & 8 & 7 & 45 \\
\hline 65 & 99 & 24 & 21 & 130 & 38 & - & 8 & 47 \\
\hline 70 & 102 & 25 & 22 & 133 & 39 & 9 & 9 & 48 \\
\hline 75 & 104 & 26 & 24 & 137 & 40 & - & 10 & 49 \\
\hline 80 & 106 & - & 26 & 141 & 41 & 10 & 12 & 51 \\
\hline 85 & 109 & 27 & 28 & 145 & 42 & 11 & 13 & 53 \\
\hline 90 & 111 & 28 & 29 & 149 & 44 & 13 & 15 & 56 \\
\hline 95 & 116 & 29 & 33 & 156 & 47 & 15 & 17 & 58 \\
\hline 99 & 121 & 30 & 36 & 166 & 49 & 20 & 21 & 65 \\
\hline
\end{tabular}


Posteriormente se procedió a realizar el análisis inferencial de los datos recolectados, obteniéndose los resultados que se detallan a continuación.

\section{Verificación de las hipótesis}

$\mathrm{H}_{1}$ : La comparación de la puntuación promedio en lectura de estudiantes de 3 . $^{\text {er }}$ grado de primaria residentes en Lima Metropolitana mostrará diferencias significativas según la variable género.

Tabla N. ${ }^{\circ}$ 21. Comparación del nivel de lectura de los estudiantes del 3.er grado de educación primaria residentes en Lima Metropolitana, considerando la variable género (global).

\begin{tabular}{cccccc}
\hline Género & N & Media & DS & t & sig \\
\hline Masculino & 129 & 128.40 & 21.034 & & \multirow{2}{*}{0.648} \\
Femenino & 148 & 129.47 & 18.264 & -.456 & \\
\hline
\end{tabular}

$\mathrm{h}_{1}$ : La puntuación promedio del nivel de procesamiento léxico de la lectura en estudiantes

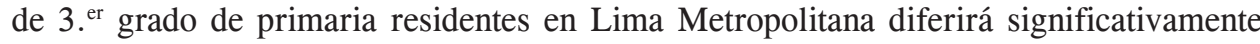
según la variable género.

Tabla N. ${ }^{\circ} 22$. Comparación del nivel de procesamiento léxico de los estudiantes del 3 er $^{\text {er }}$ grado de educación primaria residentes en Lima Metropolitana considerando la variable género.

\begin{tabular}{cccccc}
\hline Género & $\mathbf{N}$ & Media & DS & t & sig \\
\hline Masculino & 129 & 97.29 & 14.316 & & \\
Femenino & 148 & 97.64 & 12.091 & -.224 & 0.823 \\
\hline
\end{tabular}

Decisión:

La puntuación promedio del nivel de procesamiento léxico de la lectura en estudiantes

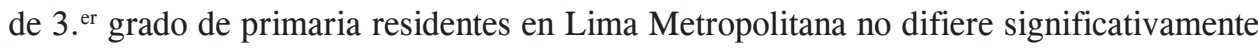
según la variable género.

$\mathrm{h}_{2}$ : La puntuación promedio de los niveles de procesamiento sintáctico-semántico de lectura de oraciones en estudiantes de 3 er $^{\text {er }}$ grado de primaria residentes en Lima Metropolitana diferirá significativamente según la variable género.

Tabla N. ${ }^{\circ}$ 23. Comparación del nivel de procesamiento sintáctico-semántico (lectura de oraciones) de los estudiantes del 3.er grado de educación primaria residentes en Lima Metropolitana considerando la variable género.

\begin{tabular}{cccccc}
\hline Género & $\mathbf{N}$ & Media & DS & t & sig \\
\hline Masculino & 132 & 23.03 & 5.983 & & \multirow{2}{*}{0.275} \\
Femenino & 148 & 23.78 & 5.552 & & \\
\hline
\end{tabular}




\section{Decisión:}

La puntuación promedio de los niveles de procesamiento sintáctico-semántico de lectura de oraciones en estudiantes de 3 er $^{\text {er }}$ grado de primaria residentes en Lima Metropolitana no difiere significativamente según la variable género.

$\mathrm{h}_{3}$ : La puntuación promedio de los niveles de procesamiento sintáctico-semántico de lectura de texto en estudiantes de 3 er $^{\text {er }}$ rado de primaria residentes en Lima Metropolitana diferirá significativamente según la variable género.

Tabla N. ${ }^{\circ}$ 24. Comparación del nivel de procesamiento sintáctico-semántico (lectura de textos) de los estudiantes del 3. ${ }^{\text {er }}$ grado de educación primaria residentes en Lima Metropolitana considerando la variable género.

\begin{tabular}{cccccc}
\hline Sexo & $\mathbf{N}$ & Media & DS & t & sig \\
\hline Masculino & 132 & 21.11 & 8.177 & \multirow{2}{*}{028} & 0.978 \\
Femenino & 148 & 21.09 & 7.465 & & \\
\hline
\end{tabular}

\section{Decisión:}

La puntuación promedio de los niveles de procesamiento sintáctico-semántico de lectura de texto en estudiantes de 3 er $^{\text {er }}$ grado de primaria residentes en Lima Metropolitana no difiere significativamente según la variable género.

$\mathrm{H}_{2}$ : La comparación de la puntuación promedio en lectura de estudiantes de $3{ }^{\text {er }}$ grado de primaria residentes en Lima Metropolitana mostrará diferencias significativas según la variable nivel socioeconómico.

Tabla N. ${ }^{0} 25$. Comparación de la puntuación promedio del nivel de lectura (global) de estudiantes de $3 .{ }^{\text {er }}$ grado de primaria residentes en Lima Metropolitana considerando la variable nivel socioeconómico.

\begin{tabular}{ccccc}
\hline NSE & N & Media (rango) & Chi cuadrado & Sig. \\
\hline NSE A & 66 & 128.42 & & \\
NSE B & 44 & 115.67 & & \\
NSE C & 47 & 153.81 & 9.024 & 0.060 \\
NSE D & 80 & 153.14 & & \\
NSE E & 40 & 136.44 & & \\
Total & 277 & & & \\
\hline
\end{tabular}

$\mathrm{h}_{1}$ : La puntuación promedio del nivel de procesamiento léxico de la lectura en estudiantes

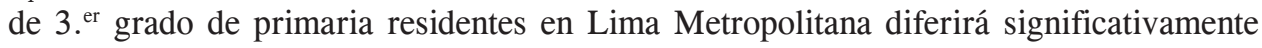
según la variable nivel socioeconómico. 
Tabla N. ${ }^{\circ}$ 26. Comparación de la puntuación promedio del nivel de procesamiento léxico de estudiantes de $3 .^{\text {er }}$ grado de primaria residentes en Lima Metropolitana considerando la variable nivel socioeconómico.

\begin{tabular}{ccccc}
\hline NSE & N & Media (rango) & Chi cuadrado & Sig. \\
\hline NSE A & 66 & 112.05 & & \\
NSE B & 44 & 109.67 & & \\
NSE C & 47 & 164.65 & 24.647 & $0.000^{*}$ \\
NSE D & 80 & 161.73 & & \\
NSE E & 40 & 140.14 & & \\
Total & 277 & & & \\
\hline
\end{tabular}

\section{Decisión:}

La puntuación promedio del nivel de procesamiento léxico de la lectura en estudiantes de 3. ${ }^{\text {er }}$ grado de primaria residentes en Lima Metropolitana difiere significativamente según la variable nivel socioeconómico.

$\mathrm{h}_{2}$ : La puntuación promedio de los niveles de procesamiento sintáctico-semántico de lectura

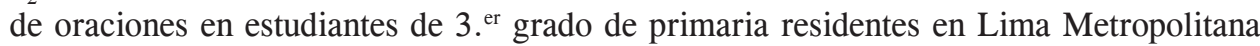
diferirá significativamente según la variable nivel socioeconómico.

Tabla N. ${ }^{\circ}$ 27. Comparación de la puntuación promedio del nivel de procesamiento sintácticosemántico (lectura de oraciones) de estudiantes de 3 er $^{\text {er }}$ grado de primaria residentes en Lima Metropolitana considerando la variable nivel socioeconómico.

\begin{tabular}{ccccc}
\hline NSE & N & Media (rango) & Chi cuadrado & Sig. \\
\hline NSE A & 67 & 156.31 & & \\
NSE B & 46 & 136.35 & & \\
NSE C & 47 & 133.33 & 5.506 & 0.239 \\
NSE D & 80 & 143.58 & & \\
NSE E & 40 & 121.06 & & \\
Total & 280 & & & \\
\hline
\end{tabular}

Decisión:

La puntuación promedio de los niveles de procesamiento sintáctico-semántico de lectura de oraciones en estudiantes de 3 . $^{\text {er }}$ grado de primaria residentes en Lima Metropolitana no difiere significativamente según la variable nivel socioeconómico.

$\mathrm{h}_{3}$ : La puntuación promedio de los niveles de procesamiento sintáctico-semántico de lectura de texto en estudiantes de 3 er $^{\text {er }}$ grado de primaria residentes en Lima Metropolitana diferirá significativamente según la variable nivel socioeconómico. 
Tabla N. ${ }^{\circ}$ 28. Comparación de la puntuación promedio del nivel de procesamiento sintácticosemántico (lectura de textos) de estudiantes de 3 . $^{\text {er }}$ grado de primaria residentes en Lima Metropolitana considerando la variable nivel socioeconómico.

\begin{tabular}{ccccc}
\hline NSE & N & Media (rango) & Chi cuadrado & Sig. \\
\hline NSE A & 67 & 169.72 & & \\
NSE B & 46 & 140.47 & & \\
NSE C & 47 & 141.86 & 16.982 & $0.002^{*}$ \\
NSE D & 80 & 132.75 & & \\
NSE E & 40 & 105.49 & & \\
Total & 280 & & & \\
\hline
\end{tabular}

\section{Decisión:}

La puntuación promedio de los niveles de procesamiento sintáctico-semántico de lectura de texto en estudiantes de 3 er $^{\text {er }}$ grado de primaria residentes en Lima Metropolitana difiere significativamente según la variable nivel socioeconómico.

$\mathrm{h}_{3}$ : La comparación de la puntuación promedio en lectura de estudiantes de 3 er $^{\text {er }}$ grado de primaria residentes en Lima Metropolitana mostrará diferencias significativas con respecto a la variable gestión de la institución educativa a la que asisten.

Tabla N. ${ }^{\circ}$ 29. Comparación nivel de lectura (global) en estudiantes de $3 .^{\text {er }}$ grado de primaria residentes en Lima Metropolitana considerando la variable gestión de la institución educativa a la que asisten.

\begin{tabular}{cccccc}
\hline Gestión & N & Media & DS & t & Sig. \\
\hline Público & 80 & 126.01 & 18.710 & & \multirow{2}{*}{0.109} \\
Privado & 197 & 130.17 & 19.834 & & \\
\hline
\end{tabular}

$\mathrm{h}_{1}$ : La puntuación promedio del nivel de procesamiento léxico de la lectura en estudiantes de 3. ${ }^{\text {er }}$ grado de primaria residentes en Lima Metropolitana diferirá significativamente respecto a la variable gestión de la institución educativa a la que asisten.

Tabla N. ${ }^{\circ} 30$. Puntuación promedio del nivel de procesamiento léxico de la lectura en estudiantes de 3 er $^{\text {er }}$ grado de primaria residentes en Lima Metropolitana considerando la variable gestión de la institución educativa a la que asisten.

\begin{tabular}{cccccc}
\hline Gestión & N & Media & DS & t & Sig. \\
\hline Público & 80 & 97.60 & 12.169 & & \\
Privado & 197 & 97.43 & 13.558 & .099 & 0.921 \\
\hline
\end{tabular}


Decisión:

La puntuación promedio del nivel de procesamiento léxico de la lectura en estudiantes de 3. ${ }^{\text {er }}$ grado de primaria residentes en Lima Metropolitana no difiere significativamente respecto a la variable gestión de la institución educativa a la que asisten.

$\mathrm{h}_{2}$ : La puntuación promedio de los niveles de procesamiento sintáctico-semántico de lectura de oraciones en estudiantes de $3 .{ }^{\text {er }}$ grado de primaria residentes en Lima Metropolitana diferirá significativamente según la variable gestión de la institución educativa a la que asisten.

Tabla N. ${ }^{0} 31$. Puntuación promedio de los niveles de procesamiento sintáctico-semántico de lectura de oraciones en estudiantes de 3.er grado de primaria residentes en Lima Metropolitana según la variable gestión de la institución educativa a la que asisten.

\begin{tabular}{cccccc}
\hline Gestión & N & Media & DS & t & Sig. \\
\hline Público & 80 & 21.84 & 6.723 & & \\
Privado & 200 & 24.07 & 5.212 & -2.963 & $0.003^{*}$ \\
\hline
\end{tabular}

\section{Decisión:}

La puntuación promedio de los niveles de procesamiento sintáctico-semántico de lectura de oraciones en estudiantes de 3. ${ }^{\text {er }}$ grado de primaria residentes en Lima Metropolitana difiere significativamente según la variable gestión de la institución educativa a la que asisten. Estas diferencias son a favor de la muestra proveniente de instituciones privadas.

$\mathrm{h}_{3}$ : La puntuación promedio de los niveles de procesamiento sintáctico-semántico de lectura

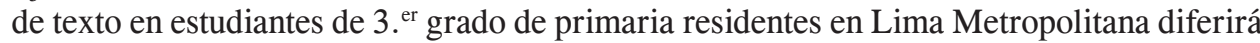
significativamente según la variable gestión de la institución educativa a la que asisten.

Tabla N. ${ }^{0} 32$. Puntuación promedio de los niveles de procesamiento sintáctico-semántico de lectura de texto en estudiantes de $3{ }^{\text {er }}$ grado de primaria residentes en Lima Metropolitana considerando la variable gestión de la institución educativa a la que asisten.

\begin{tabular}{cccccc}
\hline Gestión & N & Media & DS & t & Sig. \\
\hline Público & 80 & 17.63 & 6.830 & & \\
Privado & 200 & 22.49 & 7.738 & & $0.000^{*}$ \\
\hline
\end{tabular}

\section{Decisión:}

La puntuación promedio de los niveles de procesamiento sintáctico-semántico de lectura de texto en estudiantes de $3 .{ }^{\text {er }}$ grado de primaria residentes en Lima Metropolitana difiere significativamente según la variable gestión de la institución educativa a la que asisten. Estas diferencias son a favor de la muestra proveniente de instituciones privadas. 
$\mathrm{h}_{4}$ : El rendimiento en lectura de niños y niñas del 3. ${ }^{\text {er }}$ grado de primaria de Lima mostrará diferencias significativas con respecto al de sus pares de Piura.

Tabla N. ${ }^{\circ}$ 33. Comparación del nivel de rendimiento en lectura (Global) de niños y niñas del 3. er $^{\text {en }}$ grado de primaria de Lima respecto a sus pares de Piura.

\begin{tabular}{cccccc}
\hline Ciudad & N & Media & DS & t & Sig. \\
\hline Lima & 277 & 128.97 & 19.574 & & \\
Piura & 275 & 109.69 & 23.273 & 10.536 & $0.000^{*}$ \\
\hline
\end{tabular}

$\mathrm{h}_{1}$ : El nivel léxico de los niños y niñas del $3{ }^{\text {er }}$ grado de Lima Metropolitana mostrarán diferencias significativas con respecto al nivel presentado por sus pares de Piura.

Tabla N. ${ }^{\circ}$ 34. Comparación del nivel de procesamiento léxico de niños y niñas del $3{ }^{\text {er }}$ grado de primaria de Lima Metropolitana con respecto a sus pares de Piura.

\begin{tabular}{cccccc}
\hline Ciudad & N & Media & DS & t & Sig. \\
\hline Lima & 277 & 97.48 & 13.151 & & \multirow{2}{*}{7.088} \\
Piura & 275 & 89.05 & 14.738 & & $0.000^{*}$ \\
\hline
\end{tabular}

\section{Decisión:}

El nivel léxico de los niños y niñas del 3. ${ }^{\text {er }}$ grado de Lima muestra diferencias significativas con respecto al nivel logrado por sus pares de Piura. Estas diferencias son a favor de la muestra limeña.

$\mathrm{h}_{2}$ : Los niveles sintáctico-semántico en lectura de oraciones de los niños y niñas del 3 . er grado de Lima Metropolitana mostrarán diferencias significativas con respecto al nivel presentado por sus pares de Piura.

Tabla N. ${ }^{\circ} 35$. Comparación del nivel de procesamiento sintáctico-semántico (lectura de oraciones) de

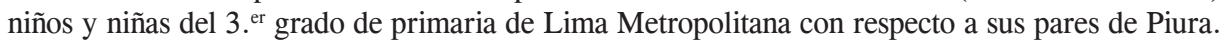

\begin{tabular}{cccccc}
\hline Ciudad & N & Media & DS & t & Sig. \\
\hline Lima & 280 & 23.43 & 5.761 & 13.183 & $0.000^{*}$ \\
Piura & 279 & 16.42 & 6.765 & & \\
\hline
\end{tabular}

\section{Decisión:}

Los niveles sintáctico-semántico en lectura de oraciones de los niños y niñas del $3 .{ }^{\text {er }}$ grado de Lima Metropolitana muestran diferencias significativas con respecto al nivel logrado por sus pares de Piura. Estas diferencias son a favor de la muestra limeña. 
$\mathrm{h}_{3}$ : Los niveles sintáctico-semántico en lectura de textos de los niños y niñas del 3 . $^{\text {er }}$ grado de Lima mostrarán diferencias significativas con respecto al nivel presentado por sus pares de Piura.

Tabla N. ${ }^{\circ}$ 36. Comparación de los niveles procesamiento sintáctico-semántico (lectura de textos) de los niños y niñas del 3 er $^{\text {er }}$ grado de Lima Metropolitana con respecto a sus pares de Piura.

\begin{tabular}{cccccc}
\hline Ciudad & N & Media & DS & t & Sig. \\
\hline Lima & 280 & 21.10 & 7.795 & \multirow{2}{*}{11.375} & $0.000^{*}$ \\
Piura & 279 & 13.29 & 8.419 & & \\
\hline
\end{tabular}

Decisión:

Los niveles sintáctico-semántico en lectura de textos de los niños y niñas del 3 er $^{\text {er }}$ rado de Lima Metropolitana muestran diferencias significativas con respecto al nivel logrado por sus pares de Piura. Estas diferencias son a favor de la muestra limeña.

$\mathrm{h}_{5}$ : El rendimiento en escritura de niños y niñas del $3 .^{\text {er }}$ grado de primaria de Lima Metropolitana mostrará diferencias significativas con respecto al alcanzado por sus pares de Piura.

Tabla N. ${ }^{\circ} 37$. Comparación del rendimiento en escritura (global) de niños y niñas del 3 er $^{\text {erado }}$ de primaria de Lima Metropolitana con respecto sus pares de Piura.

\begin{tabular}{cccccc}
\hline Ciudad & N & Media & DS & t & Sig. \\
\hline Lima & 278 & 46.08 & 10.319 & \multirow{2}{*}{0.684} & $0.000^{*}$ \\
Piura & 279 & 36.15 & 11.566 & & \\
\hline
\end{tabular}

$\mathrm{h}_{1}$ : El nivel léxico de los niños y niñas del 3. ${ }^{\text {er }}$ grado de Lima mostrará diferencias significativas con respecto al nivel logrado por sus pares de Piura.

Tabla N. ${ }^{\circ} 38$. Comparación del nivel de procesamiento léxico de los niños y niñas del $3{ }^{\text {er }}$ grado de Lima con respecto a sus pares de Piura.

\begin{tabular}{cccccc}
\hline Ciudad & N & Media & DS & t & Sig. \\
\hline Lima & 280 & 36.87 & 7.863 & \multirow{2}{*}{9.268} & $0.000^{*}$ \\
Piura & 279 & 30.04 & 9.475 & & \\
\hline
\end{tabular}

\section{Decisión:}

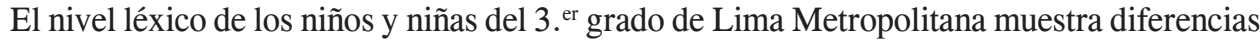
significativas con respecto al nivel logrado por sus pares de Piura. Estas diferencias son a favor de la muestra limeña. 
$\mathrm{h}_{2}$ : Los niveles sintáctico-semántico en escritura de oraciones de los niños y niñas del 3 . er grado de Lima Metropolitana mostrarán diferencias significativas con respecto al nivel logrado por sus pares de Piura.

Tabla N. ${ }^{\circ} 39$. Comparación del nivel de procesamiento sintáctico-semántico (escritura de oraciones) de los niños y niñas del $3{ }^{\text {er }}$ grado de Lima Metropolitana con respecto a sus pares de Piura.

\begin{tabular}{cccccc}
\hline Ciudad & N & Media & DS & t & Sig. \\
\hline Lima & 278 & 9.28 & 3.637 & 10.220 & $0.000^{*}$ \\
Piura & 279 & 6.16 & 3.558 & & \\
\hline
\end{tabular}

\section{Decisión:}

Los niveles sintáctico-semántico en escritura de oraciones de los niños y niñas del 3 . $^{\text {er }}$ grado de Lima Metropolitana muestran diferencias significativas con respecto al nivel logrado por sus pares de Piura. Estas diferencias son a favor de la muestra limeña.

$\mathrm{h}_{3}$ : Los niveles sintáctico-semántico de estrategias en escritura de textos de los niños y niñas del 3. ${ }^{\text {er }}$ grado de Lima Metropolitana mostrarán diferencias significativas con respecto al nivel logrado por sus pares de Piura.

Tabla N. ${ }^{\circ}$ 40. Nivel de procesamiento sintáctico semántico (escritura de textos) de los niños y niñas del $3{ }^{\text {er }}$ grado de Lima Metropolitana con respecto a sus pares de Piura.

\begin{tabular}{cccccc}
\hline Ciudad & N & Media & DS & t & Sig. \\
\hline Lima & 280 & 8.78 & 5.215 & 10.537 & $0.000^{*}$ \\
Piura & 279 & 4.27 & 5.096 & & \\
\hline
\end{tabular}

\section{Decisión:}

Los niveles sintáctico-semántico de estrategias en escritura de textos de los niños y niñas del 3. ${ }^{\text {er }}$ grado de Lima muestran diferencias significativas con respecto al nivel logrado por sus pares de Piura. Estas diferencias son a favor de la muestra limeña.

\section{DISCUSIÓN}

El estudio de los procesos cognitivos que subyacen la lectura y la escritura no es reciente. Desde la perspectiva de la psicolingüística cognitiva, la lectura se constituye en una actividad sumamente compleja que trae como corolario la elaboración de una representación mental de aquello que el autor quiso decir al escribir el texto, eso implica que no es posible reducirla a la simple percepción de las grafías, en la medida en que lo esencial de su naturaleza está referido a la transformación de símbolos lingüísticos en significados, recorriendo para ello un camino que va del lenguaje al pensamiento, teniendo como componentes a una serie de 
procesos inferidos: el perceptivo, el léxico, el sintáctico y el semántico (Defior, 2000). Sin embargo, en todo este derrotero lo único que se visualiza con exactitud es el punto de partida, el mismo que está constituido por un conjunto de grafías que deben llevar al final, mediante la serie de procesos ya mencionados, a una representación mental de lo que el autor quiso decir.

En el caso de la escritura, la situación es relativamente diferente ya que el sujeto puede escribir a la copia, al dictado o produciendo un texto de manera espontánea, este último tipo de escritura ha sido el interés del estudio, apreciándose — como en el caso de la lecturauna serie de etapas o subprocesos, los mismos que según Hayes y Flower (Cuetos, 1991) son cuatro: planificación, estructuración sintáctica, acceso al léxico y el proceso motor; de allí que este trabajo ha apuntado a caracterizar la lectura y escritura del grupo estudiado considerando estos procesos.

Sin embargo, la investigación no solo buscó replicar datos ya existentes en cuanto a los procesos que subyacen la lectura y escritura, sino explorar la relación de estos con algunos factores inherentes al individuo, como su género, y externos al mismo, entre ellos, el nivel socioeconómico al que pertenecían los alumnos, la manera en que se gestiona el proceso de enseñanza-aprendizaje en las escuelas y la localidad de procedencia de los estudiantes, asumiendo que todos ellos, de una u otra forma, inciden en la adquisición y consolidación de estos mecanismos.

Con respecto a los procesos, la observación del desempeño al efectuar la recolección de los datos mostró que los alumnos no tenían mayores inconvenientes para el reconocimiento perceptivo-visual de las palabras a las que tenían que enfrentar, reconociéndolas como tales; sin embargo, conforme transitaba el examen hacia los diferentes procesos, sus limitaciones se hicieron evidentes, de lo cual se desprende que la lectura no puede ser entendida simplemente como la adquisición y reconocimento de códigos gráficos, sino que involucra el desarrollo de una capacidad de elaboración y uso de la lengua escrita. En ese sentido se coincide con Colomer y Camps (Molina, 1998), quienes afirman que leer es algo más que un simple hecho de reconocimiento y descifrado mecánico (conversión grafema-fonema), es un acto de razonamiento que conduce al individuo a una construcción activa y reflexiva de la interpretación del mensaje escrito (Vieiro, 2004).

En cuanto al segundo aspecto, cuando se efectuó el análisis del desempeño en los diversos procesos cognitivos vinculados a la lectura y escritura, considerando la variable género, no se encontraron diferencias significativas, estos datos coinciden con lo mencionado por Bianka Zazo (Molina, 1998: 78), quien al examinar diversas habilidades neuropsicológicas encontró que niños y niñas se desarrollaban de manera semejante; en ese sentido es importante recordar que el género se refiere a nuestro sexo biológico, masculino o femenino, mientras que los roles de género, sin las expresiones externas de masculinidad o femineidad en escenarios sociales como la forma en que actuamos y pensamos como hombres y mujeres, son nuestros roles sexuales. Los roles sexuales son moldeados por tres influencias importantes, biológicas, cognitivas y ambientales (Rice, 1977: 308), de tal manera que el género en sí, no es un elemento que genere diferencias.

Sin embargo, es importante tener en cuenta que los profesores, consciente o inconscientemente, desarrollan una relación diferente con respecto a las niñas y los niños, 
aunque ello aparentemente tampoco incide en el desarrollo de los diferentes procesos vinculados a la lectura y escritura, aunque sí en otros ámbitos. Santrock (2002) señala que en el aula de clases promedio, los niños se muestran menos interesados en aprender la lectura y escritura, reciben más instrucciones y apoyo que las niñas cuando tienen problemas con una pregunta y buscan mayor atención por parte del docente, así mismo, este último pasa más tiempo interactuando con los niños varones, además de proporcionarles mayor tiempo a responder una pregunta, incluso les brindan más ayuda para que alcancen la respuesta correcta y, aun así, obtienen notas más bajas que las mujeres.

Por su parte las niñas, según el mismo autor, son más interesadas, tienden a esperar en silencio su turno; hay que llamarles la atención menos y trabajan o juegan en silencio y por sí solas; sin embargo, con todas estas supuestas ventajas tienden a creer que tendrán menos éxito en la vida universitaria, lo que hace pensar que de ocurrir ello, no es un tema vinculado a procesos cognitivos de lectoescritura, sino a situaciones ligadas a actitudes y autoestima.

De otra parte, al analizarse el papel de la primera de las variables externas, nivel socioeconómico, con respecto al desarrollo de los diferentes procesos cognitivos relacionados a la lectura y escritura, los resultados muestran que los alumnos de segmentos socioeconómicos más favorecidos de Lima Metropolitana presentaron de manera global, un mejor desempeño en lectura, superando significativamente en dos (procesamiento léxico y procesamiento sintáctico de la lectura de texto) de los tres procesos de lectura examinados (el único proceso en el que no se observaron diferencias significativas correspondió al procesamiento sintáctico-semántico de la lectura de oraciones), apreciándose de manera contundente que los alumnos de los segmentos socioeconómicos deprimidos evidenciaron un desempeño global por debajo del nivel suficiente, lo que es un factor que incide en sus posibilidades de comprensión final de un texto y en su rendimiento escolar general. En una perspectiva genérica, estos resultados coinciden con lo ya mencionado por Molina (1998: 109), quien indicó que los diversos trabajos llevados a cabo a escala internacional demuestran sin excepción alguna que la relación entre clase social y rendimiento escolar es significativa en todos los países.

Un análisis de las razones por las que se suscitan estos resultados permite deducir que dadas las evidentes posibilidades económicas de los segmentos A y B para acceder a materiales y contextos favorables e incorporar a sus niños en sistemas de estimulación temprana sistemática, permite que desarrollen una serie de habilidades previas al aprendizaje de la lectura y escritura rápidamente, por ejemplo: lenguaje oral; desencadenando ello que todos los procesos a los que se ha hecho referencia estén mejor encaminados antes de iniciar el respectivo aprendizaje de lectura y escritura. Sin embargo, se estima que no solo los elementos mencionados son importantes, sino también la actitud que el adulto de estrato socioeconómico alto tiene con respecto a la lectura y escritura, valorándola aparentemente mucho más, de allí que el aprestamiento precedente en estos segmentos no solo se limita a relacionarlo con los diversos tipos de materiales impresos, entre los cuales se encuentran los libros, siguiendo la normatividad establecida en la estructura curricular, sino que el adulto, padres o abuelos, transmiten un sentimiento de afecto y necesidad por la lectura y escritura, todo ello, dentro de una relación empática, lo que desencadena en el niño, un vínculo temprano y significativo con la lectoescritura, el mismo que alcanzará su máxima 
expresión cuando llegue a la escuela y encuentre en los textos, estructuras sintácticas, palabras y significados que ya experimentó afectivamente en el hogar de manera oral.

Ello permite deducir que los niños de niveles socioeconómicos bajos, frecuentemente faltos de oportunidades para interactuar con los adultos, dado que estos últimos no son conscientes de la importancia de este vínculo, no adquieren en sus primeros años, a nivel oral, las habilidades de composición y comprensión que necesitan para enfrentar con éxito el aprendizaje formal de la lectura y escritura.

Adicionalmente a lo mencionado es importante precisar que la experiencia muestra que los niños de nivel socioeconómico alto, en su mayoría, han experimentado desde tierna edad una apropiada relación con el texto, y por ende, con los procesos que subyacen su lectura; así como con el adulto, asumiendo un rol activo, aprendiendo a responder a interrogantes que se le plantean con respecto a lo que es leído, dialogar sobre ello y comentar sus apreciaciones; situación que no ocurre en los preescolares de nivel socioeconómico bajo, donde la relación que tienen los adultos con el texto se limita a la lectura de periódicos, folletos y material poco estructurado, mientras que a los niños se les fustiga a conservar el silencio y mantener una actitud pasiva al narrárseles una historia, limitando así el desarrollo de las habilidades lingüísticas necesarias para un adecuado aprendizaje de la lectura y escritura.

En cuanto al análisis de los procesos de lecura y escritura de los alumnos en función de la variable institución educativa de procedencia, los resultados indicaron que los estudiantes de colegios privados obtuvieron, mayoritariamente, un mejor desempeño en los diferentes procesos cognitivos examinados, lo cual refuerza la idea de que en nuestro país, la institución educativa pública se encuentra pasando por una crisis severa en cuanto a la formación que se le brinda a los estudiantes, la misma que, a decir de los diferentes agentes vinculados, involucra inadecuadas políticas de estado, falta de capacitación, actualización y perfeccionamiento de los docentes, y una inadecuada gestión de los escasos recursos económicos que se asignan al sector (Dioses, 2003).

Pero además, el conveniente desarrollo de los procesos que subyacen lectura y escritura implica la existencia de una correcta acción tutorial y la adecuada relación docente-niño $\mathrm{y}$, en ese sentido, el colegio privado funciona mejor en la medida en que existen planes y programas diseñados explícitamente para ello, además de una capacitación constante que les permite poseer una gama de estrategias y alternativas para enseñar lectura y escritura, así como reforzar los procesos subyacentes, lo que permite que el docente de la institución privada maneje adecuadamente el enfoque cognitivo apuntando a la enseñanza de la comprensión lectora y de sus complejos procesos, de tal manera que el alumno construya sus aprendizajes usando sus recursos lingüísticos ya entrenados y teniendo en cuenta los conocimientos previos que están en él mismo.

Finalmente, en cuanto al análisis considerando la localidad de procedencia de los estudiantes, los resultados han indicado un mejor desempeño de los alumnos limeños con respecto a sus pares de Piura en casi todos los procesos examinados, lo cual refuerza la tesis de que la formación de los estudiantes de provincias, en sus diversos niveles y modalidades, es inferior en calidad a la que reciben los estudiantes de Lima. 
Pero lo anterior no es una situación aislada, forma parte de las conductas y las actividades que tipifican los modos de comunicación que existen en cada microsociedad, las expectativas como grupo y las actitudes que tiene la comunidad con respecto a la lectura y escritura, haciéndose evidente que en un país con escaso desarrollo de sus diversas regiones la lectura y escritura no sea valorada con igual intensidad por todas la personas. Por ello, es altamente probable que las personas de diferentes regiones muestren distintas formas de visualizar las habilidades de lectura y escritura, haciéndose palpable el desnivel de dominio de sus procesos y la manera de aplicar estas habilidades, ya sea con fines de orden instrumental o simbólico.

\section{CONCLUSIONES}

1. No existen diferencias significativas en los procesos de lectura y escritura de los alumnos de Lima Metropolitana considerando la variable género.

2. El desempeño en los procesos que subyacen la lectura y escritura de la muestra estudiada difiere significativamente considerando la variable nivel socioeconómico.

3. El desempeño en los procesos que subyacen la lectura y escritura es significativamente mejor en los alumnos que asisten a instituciones educativas privadas.

4. El rendimiento en lectura de niños y niñas del 3 . $^{\text {er }}$ grado de primaria de Lima Metropolitana muestra diferencias significativas con respecto al alcanzado por sus pares de Piura.

a) El nivel léxico de los niños y niñas del 3 er $^{\text {er }}$ rado de Lima Metropolitana muestra diferencias significativas con respecto al nivel alcanzado por sus pares de Piura. Estas diferencias son a favor de la muestra limeña.

b) Los niveles sintáctico-semántico en lectura de oraciones de los niños y niñas del 3 er $^{\text {er }}$ grado de Lima Metropolitana muestran diferencias significativas con respecto al nivel alcanzado por sus pares de Piura. Estas diferencias son a favor de la muestra limeña.

c) Los niveles sintáctico-semántico en lectura de textos de los niños y niñas del 3 . $^{\text {er }}$ grado de Lima Metropolitana muestran diferencias significativas con respecto al nivel alcanzado por sus pares de Piura. Estas diferencias son a favor de la muestra de Lima.

5. El rendimiento en escritura de niños y niñas del 3. ${ }^{\text {er }}$ grado de primaria de Lima Metropolitana muestra diferencias significativas con respecto al de sus pares de Piura.

a) El nivel léxico de la escritura de los niños y niñas del $3 .^{\text {er }}$ grado de Lima Metropolitana muestra diferencias significativas con respecto al nivel logrado por sus pares de Piura. Estas diferencias son a favor de la muestra de Lima.

b) Los niveles sintáctico-semántico en escritura de oraciones de los niños y niñas del 3. ${ }^{\text {er }}$ grado de Lima Metropolitana muestran diferencias significativas con respecto al nivel alcanzado por sus pares de Piura. Estas diferencias son a favor de la muestra limeña. 
c) Los niveles sintáctico-semántico de estrategias en escritura de textos de los niños y niñas del $3 .^{\text {er }}$ grado de Lima Metropolitana muestran diferencias significativas con respecto al nivel logrado por sus pares de Piura. Estas diferencias son a favor de la muestra de Lima.

6. El estudio psicométrico de la Batería de Evaluación Cognitiva de Lectura y EscrituraBECOLE (nivel elemental) evidenció que es una prueba válida y confiable para examinar estos aprendizajes en alumnos de $3 .{ }^{\text {er }}$ grado de primaria de colegios estatales y particulares de todos los segmentos socioeconómicos, tanto en la ciudad de Lima, como en la ciudad de Piura.

\section{REFERENCIAS BIBLIOGRÁFICAS}

1. Bereiter, C. y Scardamalia, M. (1987). The psychology of written composition. Hillsdale, N. J: Erlbaum.

2. Cuetos, F. (2006). Psicología de la lectura. Madrid: ed. Walters Kluwer.

3. Cuetos, F; Sánchez P. y Ramos, J. L. (1996). Evaluación de los procesos de escritura en niños de educación primaria. Madrid: Bordón.

4. Cuetos, F. (1991). Psicología de la escritura. Madrid: Escuela Española.

5. Cuetos, F. (1990). Psicología de la lectura. Diagnóstico y tratamiento de los trastornos de lectura. Madrid: Escuela Española.

6. Defior, S. (2000). Las dificultades de aprendizaje: un enfoque cognitivo. Málaga: ALGIBE.

7. Dioses, A. (2009). Comprensión de estructuras gramaticales lingüísticas y procesos cognitivos de lectoescritura en alumnos de quinto grado de primaria. En Revista de Psicología Universidad César Vallejo, 11, 53-65.

8. Dioses, A. (2003). Dificultades de aprendizaje: un enfoque centrado en la tarea. En Texto Universitario. Escuela Académico Profesional de Psicología UNMSM, noviembre, 2003.

9. Flower, L. y Hayes, J. (1980). The dynamics of composing: making plans and juggling constraints. En L. Gregg y E. Steinberg (Eds.), Cognitive processes in writing. Hillsdale: LEA.

10. Galve, J. (2007). Evaluación e intervención en los procesos de lectura y escritura. Madrid: EOS.

11. Galve, J. (2005). Batería de evaluación cognitiva de la lectura y escritura. Madrid: EOS.

12. García, J. y Gonzales, D. (2000). Dificultades de aprendizaje e intervención psicopedagógica (lectura y escritura), (tomo 2). Madrid: EOS.

13. Hernández, R; Fernández, C. y Baptista, P. (1999). Metodología de la Investigación. México D.F: Mc Graw Hill. 